INTERNATIONAL ASTRONOMICAL UNION

SYMPOSIUM No. 148

\title{
THE MAGELLANIC CLOUDS
}

Edited by RAYMOND HAYNES and DOUGLAS MILNE
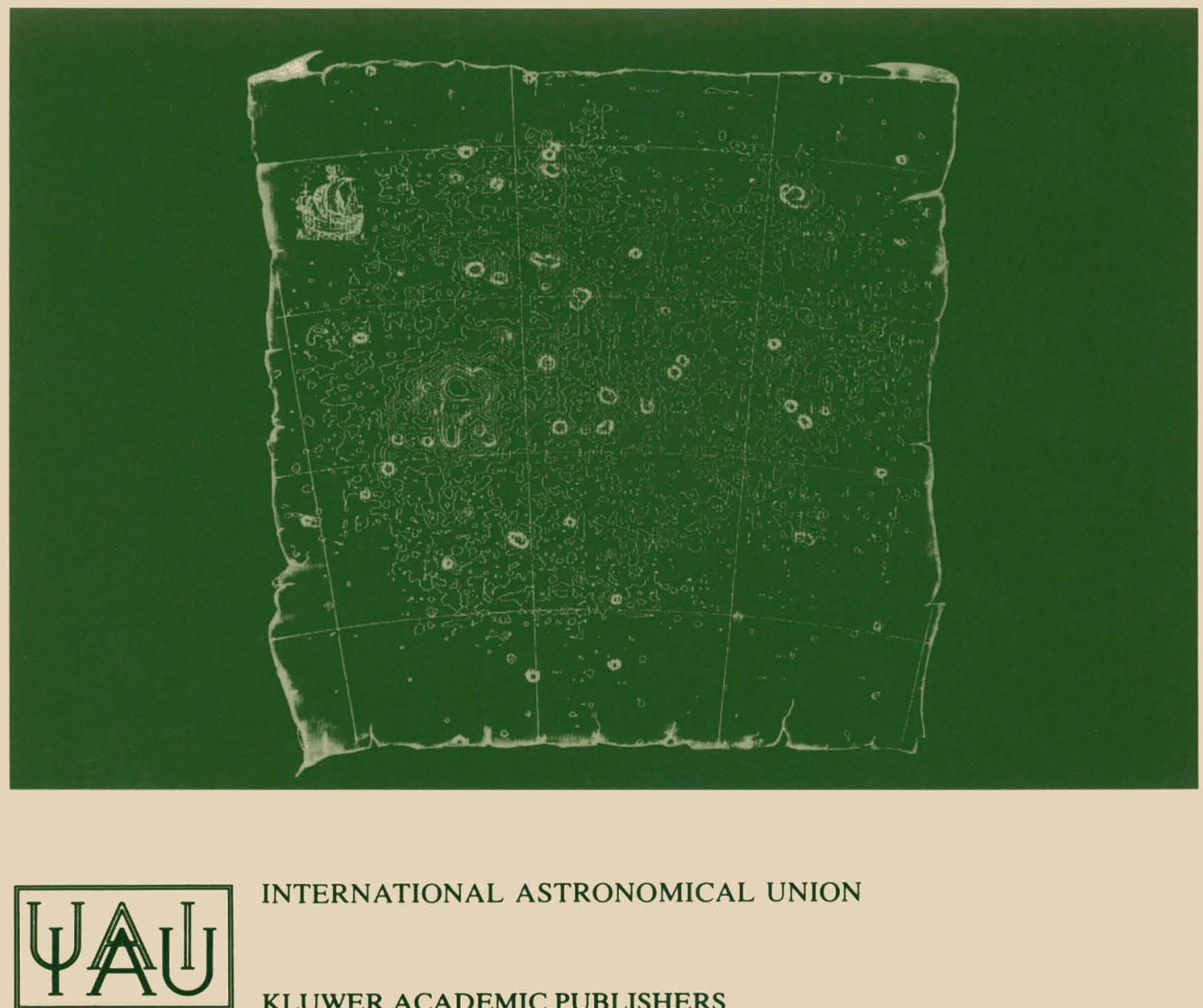

INTERNATIONAL ASTRONOMICAL UNION

KLUWER ACADEMIC PUBLISHERS 
THE MAGELLANIC CLOUDS 


\section{THE \\ MAGELLANIC CLOUDS}

PROCEEDINGS OF THE 148TH SYMPOSIUM OF THE

INTERNATIONAL ASTRONOMICAL UNION, HELD IN SYDNEY, AUSTRALIA, JULY 9-13, 1990

EDITED BY

RAYMOND HAYNES

and

DOUGLAS MILNE

Australia Telescope National Facility, CSIRO, Epping, Australia

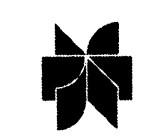

KLUWER ACADEMIC PUBLISHERS

DORDRECHT / BOSTON / LONDON

UAU 
Library of Congress Cataloging-in-Publication Data

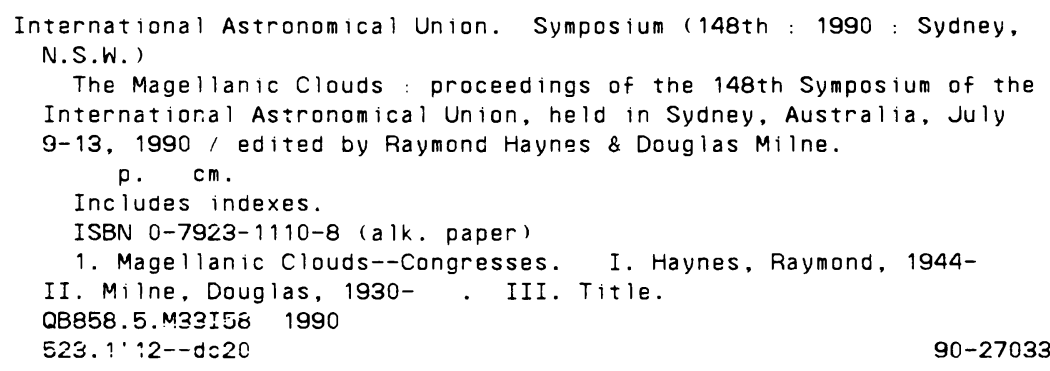

ISBN 0-7923-1110-8 (HB)

ISBN 0-7923-1111-6 (PB)

\section{Published on behalf of \\ the International Astronomical Union}

by

Kluwer Academic Publishers, P.O. Box 17, 3300 AA Dordrecht, The Netherlands.

Kluwer Academic Publishers incorporates

the publishing programmes of

D. Reidel, Martinus Nijhoff, Dr W. Junk and MTP Press.

Sold and distributed in the U.S.A. and Canada

by Kluwer Academic Publishers,

101 Philip Drive, Norwell, MA 02061, U.S.A.

In all other countries, sold and distributed

by Kluwer Academic Publishers Group,

P.O. Box 322, 3300 AH Dordrecht, The Netherlands.

Printed on acid-free paper

\section{All Rights Reserved \\ (C) 1991 International Astronomical Union}

No part of the material protected by this copyright notice may be reproduced or utilized in any form or by any means, electronic or mechanical including photocopying, recording or by any information storage and retrieval system, without written permission from the publisher.

Printed in the Netherlands 


\section{TABLE OF CONTENTS}

Preface xiii

The Organizing Committees $\quad$ xv

Group Photograph $\quad$ xvi

List of Participants $\quad$ xix

1. OPENING ADDRESS 1

The Magellanic Clouds : distance, structure, chemical composition $M W$ Feast

2. CONFERENCE SUMMARY 7

$J R$ Mould
ARGE-SCALE STRUCTURE AND KINEMATICS

$\begin{array}{ll}\text { 3. LARGE-SCALE STRUCTURE AND KINEMATICS } & 13 \\ \text { Review: An overview of the structure and kinematics of the Magellanic Clouds } & 15\end{array}$

$B E$ Westerlund

Distribution of young stars and gas in the Small Magellanic Cloud 25

$J$ Lequeux

Radio continuum emission from the Magellanic Clouds

$U$ Klein, $R F$ Haynes, $R$ Wielebinski, $S R$ Wayte, $J I$ Harnett, E Bajaja,

$J D$ Murray, A J Hunt, $R$ Wark, L Sciacca \& U R Buczilowski

An X-ray image of the Large Magellanic Cloud: detection of the hot interstellar

D J Helfand

Carbon Monoxide in the Magellanic Clouds

F P Israel \& Th de Graauw

New results on the structure and stellar content of the outer regions of the SMC

$L T$ Gardiner \& MR S Hawkins

Where is the rotation centre of the LMC?

$R$ Wielebinski \& E Bajaja

Dust in the Magellanic Clouds

Paul Hodge

HI - distribution, kinematics and geometry of the Large Magellanic Cloud

K Rohlfs \& Th Luks

Large scale distribution of gas, dust and stars deduced from observations from the

UV to the IR

J Koornneef

Large-scale structure and kinematics of the Magellanic Clouds from carbon star

$M$ Azzopardi \& E Rebeirot

Kinematics of the LMC CH-stars

F D A Hartwick \& A P Cowley 
Large scale structure and kinematics of the LMC from a study of long-period

variable stars

Shaun M G Hughes, Peter $R$ Wood \& Neill Reid

Large-scale structure of the Magellanic Clouds using planetary nebulae

89

$S J$ Meatheringham

Fast shells and X-ray emission in 30 Doradus: SNRs and superbubbles

97

You-Hua Chu \& Robert C Kennicutt Jr

$\mathrm{X}$-rays from superbubbles in the Large Magellanic Cloud

You-Hua Chu \& Mordecai-Mark Mac Low

The magnetic field strength in the Large Magellanic Cloud

101

$M E$ Costa, $P M$ McCulloch \& $P$ A Hamilton

Turbulence and stochastic star formation in the Large Magellanic Cloud

$J V$ Feitzinger \& J Spicker

A new $4 \mathrm{~m}$ millimeter-wave telescope for a southern sky CO survey

Y Fukui, H Ogawa, K Kawabata, A Mizuno \& K Sugitani

Structure and kinematics in the northeastern outer parts of the SMC

$D$ Hatzidimitriou, $R D$ Cannon \& M R S Hawkins

IR characteristics of Type II Cepheids in the LMC and in the Galaxy

107

C. David Laney

109

AST/RO and submillimetre-wave observations of the Magellanic Clouds from the

112

South Pole

$K Y$ Lo, A A Stark, $J$ Bally, $R W$ Wilson, $T$ Bania \& A Lane

The Molonglo $843 \mathrm{MHz}$ survey of the Magellanic Clouds

A J Turtle \& $S W$ Amy

The evolution of AGB stars in the Magellanic Clouds

$B E$ Westerlund, M Azzopardi, J Breysacher \& E Rebeirot

Discussion: Large-scale structure and kinematics

\section{STAR FORMATION AND CLUSTERING}

Review: Star formation in the Magellanic Clouds

A $R$ Hyland

The star formation histories of the Magellanic Clouds

HR Butcher

HII regions and star formation in the Magellanic Clouds

Robert C Kennicutt Jr

The starburst region 30 Doradus

Nolan $R$ Walborn

Recent star formation history in the region of 30 Doradus

$M-C$ Lortet, $G$ Testor \& N Martin

CO observations of the 30-Doradus region using SEST

$R S$ Booth \& L E B Johansson

Review: Star clusters in the Magellanic Clouds

$S$ van den Bergh

Magellanic globulars as cosmological tools 
The kinematics and abundances of star clusters in the Large Magellanic Cloud

$R$ A Schommer

Young star clusters in the LMC

$K C$ Freeman \& $R A W$ Elson

The age-abundance relations and age distributions for the star clusters of the

Magellanic Clouds

G S Da Costa

Mass-to-light ratios of Large Magellanic Cloud clusters

Mario Mateo, Douglas Welch \& Phil Fischer

The age distribution function of star clusters in the LMC

$M$ Alongi \& C Chiosi

A search for non-thermal emission in the HII regions of the Magellanic Clouds

$S$ W Amy

A new survey for $\mathrm{H} \alpha$-emission-line stars and related objects in the SMC

M Meyssonnier \& M Azzopardi

Similarities of the intermediate-age SMC star cluster NGC 152 with inner-disk

clusters in the Milky Way

$T$ Richtler, Klaas $S$ de Boer, A Vallenari \& W Seggewiss

The dynamics of NGC1866

Phil Fischer, D L Welch, P Coté, M Mateo \& Barry F Madore

Candidate protostars in the vicinity of 30 Doradus

202

A $R$ Hyland \& $T J$ Jones

Line observations of the 30-Dor complex and N159A5 with the MPE imaging NIR spectrometer FAST

A Krabbe, J Storey, V Rotaciuc, S Drapatz \& R Genzel

HII regions and young star clusters in the Magellanic Clouds

Myung Gyoon Lee

Observations of two different cluster types in the LMC

$P$ Linde, G Lyngå \& B E Westerlund

Dynamical study of the LMC globular cluster NGC 1978 from core velocity dispersion

G Meylan, $P$ Dubath \& M Mayor

Mass-to-light ratio of intermediate and old clusters in the Magellanic Clouds

Patrick Seitzer

Radial velocities for three distant LMC globular clusters

Jesper Storm \& Bruce W Carney

Dynamical evolution of binary clusters and colliding galaxies

D Sugimoto, $T$ Ebisuzaki \& J Makino

Properties of two star clusters of the LMC: NGC 2164, NGC 1850

A Vallenari, C Chiosi, G Bertelli, G Meylan \& S Ortolani

Effects of data incompleteness and metallicity on the mass functions of young

LMC star clusters

$T$ Richtler, $R$ Sagar, A Vallenari \& Klaas $S$ de Boer

An X-ray survey of OB associations in the Large Magellanic Cloud

Qingde Wang \& David J Helfand 
Faint HII regions in the Magellanic Clouds

Eric $M$ Wilcots \& Paul Hodge

Infrared imaging of intermediate age $\mathrm{LMC} / \mathrm{SMC}$ clusters

H Zinnecker, F Ferraro, F Fusi Pecci, Alvio Renzini, R Buonanno,

$C$ E Corsi \& D M Terndrup

A 22-GHz map of the 30-Doradus region

N S Sabalisck \& Z Abraham

N159A: a region of active star formation in the LMC

L Deharveng \& J Caplan

Shapes of Magellanic Cloud star clusters

E Kontizas, M Kontizas, G Sedmak \& R Smareglia

Discussion: Star Formation and Clustering

5. STELLAR EVOLUTION

Review: The chemical evolution of the Magellanic Clouds

$F$ Spite \& M Spite

Cepheids in the Magellanic Clouds

John A R Caldwell \& C David Laney

Long-period variables in the Magellanic Clouds

$P R$ Wood, $G K G$ Moore \& SM G Hughes

Luminous blue variable stars in the Magellanic Clouds

Bernhard Wolf

Wolf-Rayet stars in the Magellanic Clouds

Lindsey $F$ Smith

The interstellar reddening and metallicity of NGC 330

Michael S Bessell

Winds of hot stars in the Magellanic Clouds

$R P$ Kudritzki, A W A Pauldrach, J Puls \& S R Voels

Binary $X$-ray sources in the LMC

$J B$ Hutchings \& A P Cowley

Abundances in Magellanic Cloud planetary nebulae

M J Barlow

Planetary nebulae in the Magellanic Clouds

$M$ A Dopita

RR Lyrae stars in the Magellanic Clouds

Alistair $R$ Walker

Studies of SNRs in the Magellanic Clouds

A $J$ Turtle

SN 1987A and its role in the LMC

I J Danziger, $P$ Bouchet, $C$ Gouiffes \& L B Lucy

Radio pulsars in the Magellanic Clouds

$D$ McConnell, $P M$ McCulloch, $P$ A Hamilton, $J G$ Ables, P J Hall, C E Jacka, \& A J Hunt

First Australia Telescope observations of SNR 0540-693

M J Kesteven, R N Manchester \& L Staveley-Smith 
Effects of envelope overshoot on the bump of the Red Giant Branch luminosity function

$M$ Alongi, G Bertelli, A Bressan \& C Chiosi

Effects of envelope overshoot on intermediate mass stars

$M$ Alongi, G Bertelli, A Bressan \& C Chiosi

Monitoring SN1987A at $45 \mathrm{MHz}$

H Alvarez, J Aparici, J May \& F Olmos

Supernova explosions in stellar wind bubbles

S Jane Arthur

Abundances and nebular and central star masses for Magellanic Cloud planetary nebulae

$N A$ Walton, M J Barlow, D J Monk \& R E S Clegg

Search for UHE gamma ray sources in the Magellanic Clouds

I A Bond

IUE observations of stars in NGC 330 in the Small Magellanic Cloud

$V$ Caloi, A Cassatella, V Castellani \& G Klare

Metal abundances of Magellanic Cloud clusters

D Geisler, A McWilliam, N Suntzeff, M Mateo, J Graham

\& L Pasquini

Star formation histories in dwarf irregular galaxies in the Local Group

L Greggio, G Marconi, P Focardi, \& M Tosi

The spectrum of SN1987A in the LMC in July 1987

$C$ C Huang

Australia Telescope observations of the supernova remnant N49 in the LMC

Norbert Junkes

Radio imaging of the supernova remnant N132D in the Large Magellanic Cloud

John R Dickel \& D K Milne

A photometric and spectroscopic study of R Coronae Borealis stars in the LMC

$W$ A Lawson, $P$ L Cottrell \& $K R$ Pollard

Charting cool carbon stars in the Large Magellanic Cloud

$M F M c C a r t h y S J \& V M$ Blanco

A search for long period variables in the SMC

$G K G$ Moore

Two more Magellanic Cloud CM diagrams

$J R$ Mould, J B Jensen, G S Da Costa, J Kristian \& J M Nemec

Properties of fast winds in hot stars in the Magellanic Clouds

$P$ Patriarchi \& M Perinotto

Asymptotic Giant Branch Stars in the Magellanic Clouds

Neill Reid \& J R Mould

A survey for RR Lyrae variables in the Large Magellanic Cloud

Neill Reid \& Wendy L Freedman

The chemical evolution of the Magellanic Clouds

$S$ C Russell

Carbon and nitrogen in the cool supergiant A7 of the young globular cluster

NGC 330 in the SMC

B Barbuy, A Milone, $M$ Spite \& F Spite 
Comparison of the abundances in the stars and in the HII regions of the Magellanic

Clouds

$M$ Spite \& F Spite

New element abundances in NGC 330

$T$ Richtler, $M$ Spite \& $F$ Spite

Michigan 160: a precursor to the LMC?

L Staveley-Smith

N132D: a chemical and dynamic analysis

Ralph S Sutherland \& M A Dopita

First CCD observations of Magellanic Cloud variable stars from the Mt John

University Observatory, New Zealand

William Tobin, A C Gilmore, Alan Wadsworth \& SR D West

The anomalous A-type supergiants in the Magellanic clouds - evidence for

post-red supergiant evolution

$R$ M Humphreys, $R$ P Kudritzki \& H Groth

The variable star population of NGC 1866

Douglas L Welch, Patrick Coté, Mario Mateo \& Barry F Madore

$\mathrm{OH} / \mathrm{IR}$ stars in the Large Magellanic Cloud: the observations

$P R$ Wood, $M S$ Bessell, $S M G$ Hughes, A R Hyland, J B Whiteoak,

F F Gardner \& R E Otrupcek

Element abundances from B stars in the Magellanic Clouds

A Jüttner, $O$ Stahl, B Wolf \& B Baschek

EXOSAT Medium energy observations of the binary X-ray pulsar SMC X1

Zhj-Yun Zang

Abundance analysis of six LMC F supergiants

Andrew McWilliam \& Robert E Williams

Summary: Chemical abundances and chemical evolution of the Magellanic Clouds :

Prospects for the future

$M$ A Dopita

Discussion: Stellar Evolution

6. THE INTERSTELLAR MEDIUM

Review: The interstellar medium of the Magellanic Clouds from absorption lines

Klaas $S$ de Boer

Dust distribution and temperature in the Magellanic Clouds interstellar medium

$M$ Sauvage \& L Vigroux

Molecules in the Magellanic Clouds

$R S$ Booth and Th de Graauw

Studies of the Large Magellanic Cloud using optical interstellar emission lines

JMeaburn

Molecular gas in the Small Magellanic Cloud

Monica Rubio

Studies of the ISM in the LMC using SN1987A

Max Pettini

Ultra-high-energy cosmic rays in the Magellanic Clouds 
$\mathrm{NaI}$ and CaII interstellar absorption in the field of SN1987A

$P$ Molaro, $G$ Vladilo, $S D^{\prime} O$ dorico, $M$ Dennefeld, $R$ Ferlet \& A Vidal-Madjar

Depth structure in the gas in Shapley III along three lines of sight

436

Dominik J Bomans, Klaas $S$ de Boer \& Jan Koornneef

Interstellar gas around WO stars in the Galaxy and the Magellanic Clouds

438

Tatiana A Lozinskaya

Enhanced galactic star formation as caused by the Milky Way - Large Magellanic

Cloud interaction: an experience from density wave studies

L S Marochnik \& A A Suchkov

Discussion: The Interstellar Medium

441

7. THE LMC-SMC-GALAXY SYSTEM

445

Review : The interacting Magellanic System

447

$S R$ Wayte

Optical surveys of the Magellanic Bridge, Stream and outer halos

453

M J Irwin

High velocity clouds near the Magellanic Clouds

463

Ulrich Mebold

Asymmetric distribution of gas in the LMC and dynamical condition for globular

469

cluster formation

Mitsuaki Fujimoto \& Yasuki Kumai

Metallicity effects on the Cepheid distance scale

471

Wendy L Freedman \& Barry F Madore

Radial velocities of clusters between the Magellanic Clouds

473

$S$ Demers, L Grondin, M J Irwin \& W E Kunkel

Orbits of the Magellanic Clouds

Slobodan Ninkovic \& Miroslav Filipovic

String of young clusters linking the Magellanic Clouds

478

$L$ Grondin, $S$ Demers, $W$ E Kunkel \& M J Irwin

Mass loss and evolution of massive stars in the Magellanic Clouds

480

Claus Leitherer \& Norbert Langer

The Magellanic Stream

483

W L H Shuter

$\mathrm{CO}$ observations of Arp's interacting galaxies

485

Yoshiaki Sofue, Ken-ichi Wakamatsu, Yoshiaki Taniguchi, Naomasa Nakai

\&Toshihiro Handa

The effect of the Magellanic Clouds on the mass distribution in the galaxy

487

$G X$ Song

Special purpose computer for $\mathrm{N}$-body problems

489

D Sugimoto, T Ebisuzaki, T Ito \& J Makino

Measurement of the absolute proper motions of the Magellanic Clouds

H-J Tucholke \& M Hiesgen

Discussion: The LMC-SMC-Galaxy System

Author Index

Subject Index 